\title{
Saturated-absorption spectroscopy: eliminating crossover resonances by use of copropagating beams
}

\author{
Ayan Banerjee and Vasant Natarajan \\ Department of Physics, Indian Institute of Science, Bangalore, 560 012, India
}

Received May 22, 2003

\begin{abstract}
We demonstrate a new technique for saturated-absorption spectroscopy by use of copropagating beams that does not have the problem of crossover resonances. The pump beam is locked to a transition, and its absorption signal is monitored while the probe beam is scanned. As the probe comes into resonance with another transition, the pump absorption is reduced and the signal shows a Doppler-free dip. We use this technique to measure hyperfine intervals in the $D_{2}$ line of ${ }^{85} \mathrm{Rb}$ with a precision of $70 \mathrm{kHz}$ and to resolve hyperfine levels in the $D_{2}$ line of ${ }^{39} \mathrm{~K}$ that are less than $10 \mathrm{MHz}$ apart. (C) 2003 Optical Society of America

OCIS codes: $300.6460,300.6210,020.2930$.
\end{abstract}

Laser spectroscopy in atomic vapor is often limited by Doppler broadening. The standard method to overcome the first-order Doppler effect is to use saturated-absorption spectroscopy. ${ }^{1}$ In this technique the laser is split into a weak probe beam and a strong pump beam, and the two beams are sent in opposite directions through the vapor. Because of the opposite Doppler shifts, only the zero-velocity atoms (i.e., atoms moving perpendicular to the direction of the beams) interact with both beams. For these atoms, the stronger pump beam saturates the transition and the probe-absorption spectrum shows a Doppler-free dip at line center. Ideally, the linewidth of the dip is limited only by the natural linewidth of the transition.

The analysis presented above is correct for two-level atoms, i.e., where the other atomic levels are far away compared with the Doppler width. However, in most real atoms, there are several closely spaced hyperfine levels within the Doppler profile. The presence of multiple levels is a problem in saturated-absorption spectroscopy and results in what are called crossover resonances. These spurious resonances occur when the laser is tuned exactly midway between two transitions, so that for some velocity group the pump drives one transition while the probe drives the other. Since both transitions start from the same ground level, the pump is still effective in reducing absorption from the probe. Crossover resonances are particularly problematic for high-resolution spectroscopy on two levels whose spacing is only slightly larger than the natural linewidth. In this case the crossover resonance often swamps the real peaks and makes it impossible to resolve the two transitions.

In this Letter we demonstrate an alternative technique for saturated-absorption spectroscopy that does not have the problem of crossover resonances. The technique uses to advantage the multilevel structure of the atom in creating differential absorption between the pump and probe beams. The basic idea is to have the two beams copropagate through the vapor, with the pump frequency fixed on one transition while the probe is scanned over the other transitions. Unlike in conventional saturated-absorption spectroscopy, both beams have roughly equal intensities, and it is the absorption of the pump beam that is monitored. Since the pump is locked to a transition, it is absorbed only by the zero-velocity atoms. The absorption signal remains constant as the probe is scanned, since the probe is generally absorbed by a different velocity group. However, as the probe comes into resonance with another transition for the zero-velocity group, the absorption of the pump is reduced and the signal shows a Doppler-free dip.

There are two primary advantages to our scheme. The first, as mentioned above, is the absence of crossover resonances. The second is that the signal appears on a flat background. This is different from conventional saturated-absorption spectroscopy where the probe absorption has an underlying Doppler profile that can affect the determination of the peak centers. We have applied this technique to spectroscopy on the $D_{2}$ lines of ${ }^{85} \mathrm{Rb}$ and ${ }^{39} \mathrm{~K}$. In the case of $\mathrm{Rb}$, we measure the hyperfine interval between the $F=1$ and $F=2$ levels in the $5 P_{3 / 2}$ state with a precision of $70 \mathrm{kHz}$. In $\mathrm{K}$, we are able to resolve all three hyperfine transitions in the $D_{2}$ line, which are completely merged in ordinary saturated-absorption spectroscopy.

The first set of experiments were done with a vapor of ${ }^{85} \mathrm{Rb}$ atoms. The experimental schematic is shown in Fig. 1. The pump and probe beams are derived from two frequency-stabilized diode lasers $^{2}$ operating on the $\mathrm{Rb} D_{2}$ line at $780 \mathrm{~nm}$ $\left(5 S_{1 / 2} \leftrightarrow 5 P_{3 / 2}\right.$ transition). The intensity in each beam is $\sim 10 \mu \mathrm{W} / \mathrm{cm}^{2}$. The two beams copropagate through a room-temperature vapor cell such that the angle between them is less than $10 \mathrm{mrad}$. The pump laser is locked to a hyperfine transition by use of saturated-absorption spectroscopy in another $\mathrm{Rb}$ vapor cell. The probe laser is scanned by double passing it through an acousto-optic modulator $(\mathrm{AOM})$ and scanning the AOM frequency. The double passing is necessary to maintain directional stability of the beam, which can otherwise lead to systematic shifts of the peak positions. The probe intensity is also stabilized by adjustment of the rf power into the $\mathrm{AOM}$ in a servo loop.

A normal saturated-absorption spectrum for the $D_{2}$ line in ${ }^{85} \mathrm{Rb}$ is shown in Fig. 2. The underlying 


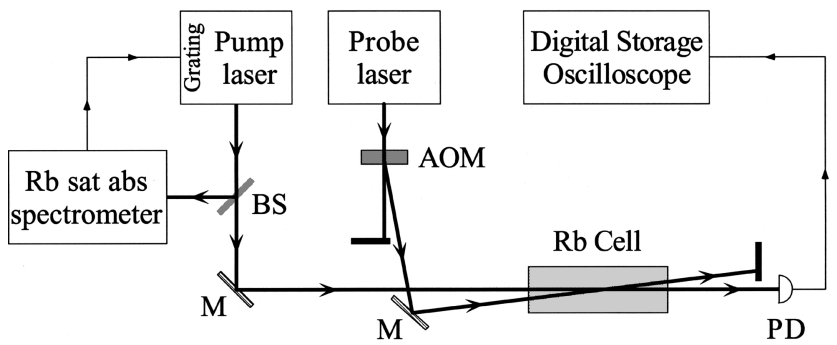

Fig. 1. Schematic of the experiment. The pump laser is locked to a hyperfine transition in $\mathrm{Rb}$, and the probe laser is scanned by double passing it through an AOM and scanning the AOM frequency. The two beams copropagate through a $\mathrm{Rb}$ vapor cell. The angle between them has been exaggerated for clarity. BS, beam splitter; Ms, mirrors; PD, photodiode.

Doppler profile has been subtracted with a second probe beam that does not interact with the pump. The spectrum is for transitions starting from the $F=2$ ground level. The $F^{\prime}=1$ and $F^{\prime}=2 \mathrm{lev-}$ els in the excited state are barely visible because they are only $30 \mathrm{MHz}$ apart ${ }^{3}$ and overlap with the crossover resonance in between. The linewidth of the peaks is $12-15 \mathrm{MHz}$, compared with the natural linewidth of $6 \mathrm{MHz}$. The primary causes for the increased linewidth are power broadening that is due to the pump beam and a small angle between the counterpropagating pump and probe beams. In addition, the line shape of the peaks depends crucially on the intensities in the two beams. At high pump intensities, optical-pumping effects and the effect of velocity redistribution of the atoms from radiation pressure leads to inversion of the peaks or distortion of their Lorentzian line shape. ${ }^{4}$ This can be seen from the lower trace of Fig. 2, where the pump intensity has been increased by a factor of 2 . The $F^{\prime}=1$ and $F^{\prime}=2$ peaks get distorted, and the $F^{\prime}=(1,2)$ crossover resonance becomes inverted.

The dramatic improvement in the spectrum with our new technique can be seen from Fig. 3. The pump laser is locked to the $F=2 \leftrightarrow F^{\prime}=3$ transition while the probe laser is scanned over a frequency range of $50 \mathrm{MHz}$ covering the $F^{\prime}=1$ and $F^{\prime}=2$ hyperfine levels. The pump-transmission signal shows two wellresolved peaks corresponding to these levels. The spectrum appears on a flat background, and there is no crossover resonance in between. The peaks also have symmetric Lorentzian line shapes with no significant pulling by the neighboring peak.

There are two advantages to scanning the probe laser with an AOM instead of the grating angle that controls the optical feedback. The first is that the use of the AOM guarantees linearity of the scan since the voltage-controlled oscillator that determines the AOM frequency has a linear transfer function. Second, the scan axis has absolute frequency calibration once the voltage-to-frequency transfer function of the voltage-controlled oscillator is known. This transfer function is readily measured with a frequency counter. We used the advantage of a calibrated frequency scan to precisely determine the hyperfine interval in Fig. 3. By fitting Lorentzians to the two peaks, we determine the peak centers with a precision of $50 \mathrm{kHz}$. This yields the value of the interval to be 29.35(7) $\mathrm{MHz}$. The result is in good agreement with earlier values of 29.26(3) $\mathrm{MHz}$ (Ref. 5) and 29.30(3) $\mathrm{MHz}$ (Ref. 6) that we obtained by use of other techniques. The high level of precision in this Letter is a direct consequence of being able to resolve the two transitions without an intervening crossover resonance. One potential source of error in hyperfine measurements is a systematic shift in the lock point of the laser from peak center. However, in our scheme, this is not a problem because both the pump and the probe beams address the same velocity group. Thus a shift in the pump-laser lock point causes the entire set of hyperfine peaks to shift, but the intervals do not change.

We performed a second set of experiments with ${ }^{39} \mathrm{~K}$. For these experiments the two diode lasers were chosen to operate on the $D_{2}$ line of $\mathrm{K}$ at $767 \mathrm{~nm}\left(4 S_{1 / 2} \leftrightarrow 4 P_{3 / 2}\right.$ transition). The probe laser did not pass through an AOM but was scanned by scanning the grating. The

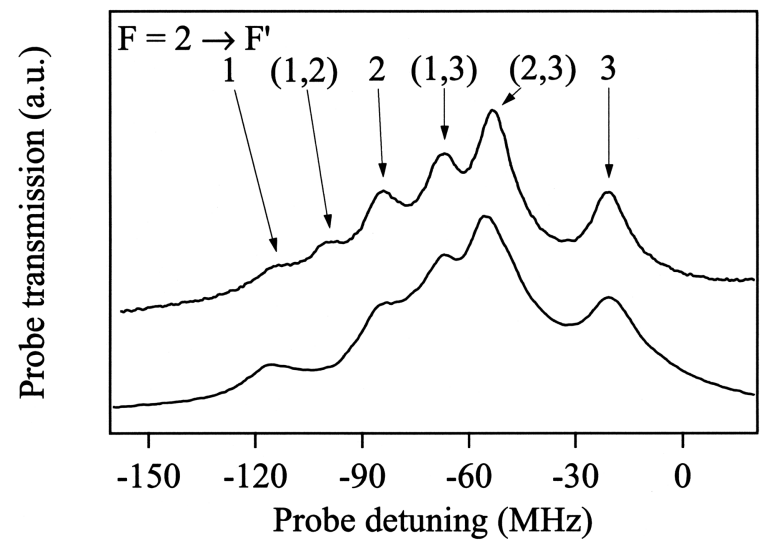

Fig. 2. Saturated-absorption spectrum in the $D_{2}$ line of ${ }^{85} \mathrm{Rb}$ for $F=2 \rightarrow F^{\prime}$ transitions. The underlying Doppler profile has been subtracted. The transitions are labeled with the value of $F^{\prime}$ and crossover resonances with the two values of $F^{\prime}$ in brackets. The upper trace is the correct spectrum, and the lower trace is obtained when the pump intensity is increased by a factor of 2 , resulting in inversion of the $F^{\prime}=(1,2)$ peak. Probe detuning is measured from the unperturbed state.

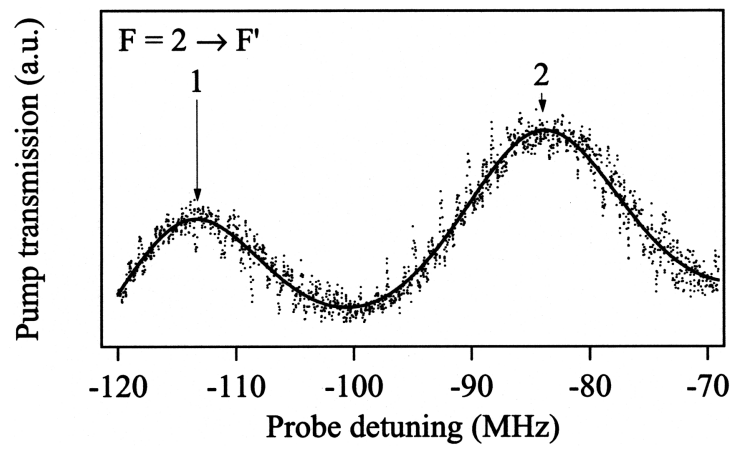

Fig. 3. ${ }^{85} \mathrm{Rb}$ spectrum obtained with new technique. The transitions are the same as in Fig. 2, with a narrow scan around the $F^{\prime}=1$ and $F^{\prime}=2$ levels. The frequency scale on the $x$ axis is set by the voltage-controlled oscillator driving the AOM. The Lorentzian fit (solid curve) yields a value of 29.35(7) $\mathrm{MHz}$ for the hyperfine interval. 


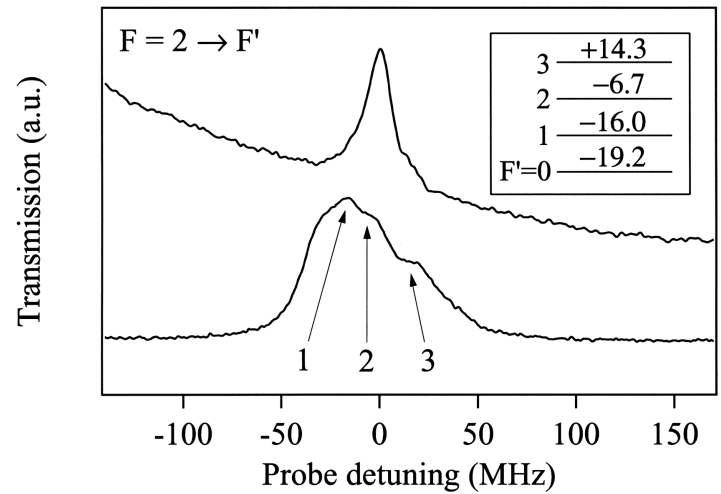

Fig. 4. Saturated-absorption spectrum in the $D_{2}$ line of ${ }^{39} \mathrm{~K}$. The top trace shows the probe-transmission signal for $F=2 \rightarrow F^{\prime}$ transitions in usual saturated-absorption spectroscopy. The six peaks are merged into a single peak since the hyperfine levels lie within $30 \mathrm{MHz}$, as shown in the inset. The values in the inset are the frequency offset (in megahertz) of each hyperfine level from the unperturbed state. The bottom trace is the pump-transmission signal obtained with the new technique, clearly showing the locations of the hyperfine levels.

consequent variation in the direction was negligible for the results presented here. The beams copropagated through an ultrahigh vacuum glass cell maintained at a pressure below $10^{-8}$ Torr by an ion pump. $\mathrm{K}$ vapor was produced by heating of a getter source ${ }^{7}$ with a current of $2.6 \mathrm{~A}$. The ultrahigh vacuum environment was necessary to minimize linewidth broadening arising as a result of background collisions. The getter source also gave us control over the amount of $K$ vapor in the cell, which we optimized to obtain the narrowest linewidth.

In Fig. 4, we show the spectrum for $F=2 \rightarrow F^{\prime}$ transitions in ${ }^{39} \mathrm{~K}$. With conventional saturatedabsorption spectroscopy, we obtain a single peak containing all the transitions. The underlying Doppler profile can also be seen. The line shape of the peak is a convolution of six peaks lying within $30 \mathrm{MHz}$ of each other. ${ }^{3}$ Indeed, it is hard to identify the location of the peak center with respect to the actual hyperfine transitions. By contrast, the new technique shows the locations of the three levels quite clearly. Although the linewidth is still large enough that the peaks overlap, there is no ambiguity in identifying their locations.

In summary, we have demonstrated a technique for saturated-absorption spectroscopy in multilevel atoms by use of copropagating pump and probe beams. The pump laser is locked to a transition so that it addresses only the zero-velocity atoms. The transmission of the pump shows Doppler-free peaks when the probe comes into resonance with another transition for the same zero-velocity atoms. The main advantages of the technique are the absence of crossover resonances and the appearance of the signal on a flat background. We demonstrate the power of this technique for hyperfine spectroscopy of closely spaced levels, using an AOM to measure the smallest interval in the $5 P_{3 / 2}$ state of ${ }^{85} \mathrm{Rb}$. We are also able to resolve hyperfine transitions in the $D_{2}$ line of ${ }^{39} \mathrm{~K}$. In the work reported here we used two lasers to generate the pump and probe beams, which appears disadvantageous compared with the use of one laser in conventional saturated-absorption spectroscopy. However, it is possible to generate the probe beam from the pump laser by use of one or more AOMs, which has advantages for scanning the probe in a calibrated manner. Finally, the pump intensity in our scheme has to be kept very small (below $\sim 10 \mu \mathrm{W} / \mathrm{cm}^{2}$ ). At higher intensities, one can see clear evidence of dressed states created as a result of the coherent driving by the pump laser. Indeed, we used this technique as a means of studying dressed states in roomtemperature vapor without complications from Doppler broadening. ${ }^{8}$

The author's thank Dipankar Das for help with the experiments. This work was supported by the Department of Science and Technology, Government of India. V. Natarajan's e-mail address is vasant@physics.iisc.ernet.in.

\section{References}

1. W. Demtröder, Laser Spectroscopy (Springer-Verlag, Berlin, 1982).

2. A. Banerjee, U. D. Rapol, A. Wasan, and V. Natarajan, Appl. Phys. Lett. 79, 2139 (2001).

3. E. Arimondo, M. Inguscio, and P. Violino, Rev. Mod. Phys. 49, 31 (1977).

4. R. Grimm and J. Mlynek, Appl. Phys. B 49, 179 (1989).

5. U. D. Rapol, A. Krishna, and V. Natarajan, Eur. Phys. J. D 23, 185 (2003).

6. A. Banerjee, D. Das, and V. Natarajan, Opt. Lett. 28, 1579 (2003).

7. SAES Getters S.p.A., Viale Italia 77-1-20020, Lainate (MI), Italy.

8. U. D. Rapol and V. Natarajan, "Doppler-free spectroscopy in driven three-level systems," Eur. Phys. J. D (to be published). 